MR-10815-177

\title{
CONTRACT STATUS REPORT FOR MHD RESEARCH AND DEVELOPMENT AND OPERATION OF THE MHD COAL FIRED FLOW FACILITY
}

For The Month Ending

May 31, 1995

Work Performed Under Contract No. DE-AC02-79ET10815

\author{
Prepared by \\ The University of Tennessee \\ Space Institute \\ Energy Conversion Research \& Development Programs
}

For

The United States Department of Energy

DISTAIBUTION OF THIS DOCUMENT IS UNLIMTED

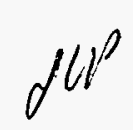




\section{DISCLAIMER}

This report was prepared as an account of work sponsored by an agency of the United States Government. Neither the United States Government nor any agency thereof, nor any of their employees, make any warranty, express or implied, or assumes any legal liability or responsibility for the accuracy, completeness, or usefulness of any information, apparatus, product, or process disclosed, or represents that its use would not infringe privately owned rights. Reference herein to any specific commercial product, process, or service by trade name, trademark, manufacturer, or otherwise does not necessarily constitute or imply its endorsement, recommendation, or favoring by the United States Government or any agency thereof. The views and opinions of authors expressed herein do not necessarily state or reflect those of the United States Government or any agency thereof. 


\section{DISCLAIMER}

\section{Portions of this document may be illegible}

in electronic image products. Images are produced from the best available original document. 


\section{PROJECT STATUS REPORT}

1. CONTRACT OBJECTIVE - No change since last report.

2. TECHNICAL APPROACH CHANGES - None

3. CONTRACT TASKS - In this report, UTSI reports on the status/progress by program tasks identified in Chapter 3 (Work Plan) of the draft Contract Management Plan for the FY95 period, which was submitted to DOE February 17, 1995.

\section{TASK I-CONTRACT MANAGEMENT AND CLOSEOUT}

The final October-December 1994 and January-March 1995 Quarterly Technical Progress Reports were issued.

Final preparations are being made for the 33rd Symposium on Engineering Aspects of Magnetohydrodynamics hosted by UTSI and ERC, Inc. June 12-16, 1995.

In response to a DOE/PETC letter of $5 / 23 / 95$, discussions and work were initiated on the development of a contract proposal package for "UTSI/CFFF MHD Program Completion and Related Activity."

Personnel medical records were returned to UTSI from the Occupational Medical Consultant (OMC) as a result of the OMC subcontract termination.

The contract status report for April 1995 was issued to DOE.

A no-cost contract extension (MOD \#M112), extending contract performance through September 30,1995, was received, signed and returned to DOE.

DOE letter authorizing purchases through GSA sources was received.

Personnel staffing for the month was 21.9 equivalent full-time (EFT) personnel versus a planned level of 26.2 EFT.

\section{TASK II - FACILITY AND PROPERTY DISPOSITION}

Facility activities this month were confined to the continuation of preventative maintenance and repairs required to maintain the Coal-Fired Flow Facility (CFFF) in a standby condition. 
The reconditioning of the CFFF process water cooling tower continued. A two week shutdown has been scheduled for this system to complete the work.

Control of government property and the maintenance of records were continuing.

Property custodians completed their review of all property items to determine if any property should be retired or placed on a surplus list if not required. Several property items are expected to be processed for disposition.

\section{TASK III-DATA REPORTING AND ARCHIVING}

Preparation of topical reports pertaining to the MHD POC test program continued.

Corrosion analyses of the Superheater Test Module tube specimens from the Western Coal POC tests were continuing.

Continued the small scale laboratory experiments to acquire fundamental data on secondary combustion in the presence of potassium compounds. This work is to be documented in a doctoral dissertation, when completed.

All data and documentation developed thus far on the MHD/CFFF project and identified for archiving are continuing to be maintained for archiving storage.

\section{TASK IV - SITE ENVIRONMENTAL COMPLIANCE AND REMEDIATION}

Under a subcontract for the first round of groundwater samples which will be used as a screening technique to verify what parameters must be analyzed in the other six wells that will be installed. This technique will save funds by eliminating all groundwater pollutants except those detected above drinking water standards.

Monitoring of the holding pond effluent continued in order to comply with the UTSI water discharge permit. Water is checked for $\mathrm{pH}$, temperature, flow rate, oil and grease content, total dissolved solids, and total suspended solids. All parameters continue to be measured by the UTSI chemistry lab. Any changes in CFFF operation might require monitoring of additional parameters.

\section{OPEN ITEMS}

A. DOE: The following reports/documents were in DOE review for comments and/or approval as of May 31, 1995.

FY1995 Management Plan

UTSI Itr 02/17/95 
B. UTSI: The following actions were in process or preparation as of May 31, 1995. Superheater/Air Heater Tube Corrosion Studies Est. Completion 09/30/95 $\mathrm{NO}_{\mathrm{x}}$ Formation and Control, Topical Report Est. Completion 06/30/95 Western Coal Processing System, Topical Report Est. Completion 06/30/95 Western Coal 1,000 Hr POC \& Final Summary Report Est. Completion 06/30/95

\section{SUMMARY STATUS ASSESSMENT AND FORECAST}

The CFFF continues in standby condition as preventive maintenance and repairs are accomplished as needed.

Efforts on the preparation of topical reports continued. 


\section{Milestone Schedule and Status Report}

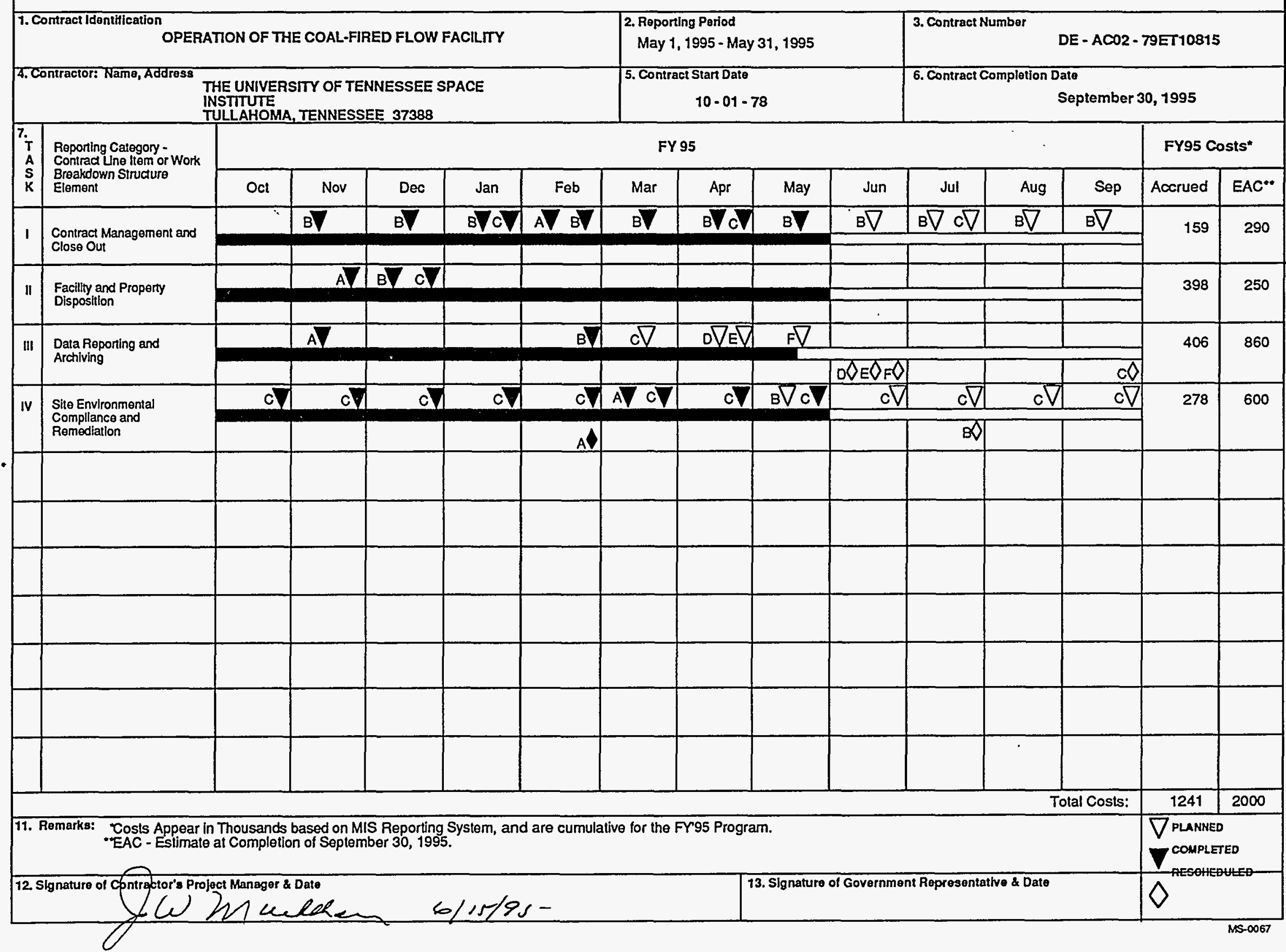


MILESTONE LOG

\begin{tabular}{|c|c|c|c|c|c|}
\hline I.D. NO. & DESCRIPTION & $\begin{array}{c}\text { ACTUAL } \\
\text { COMPLETION } \\
\text { DATE }\end{array}$ & $\begin{array}{c}\text { PLANNED } \\
\text { COMPLETION } \\
\text { DATE }\end{array}$ & $\begin{array}{c}\text { EXPECTED } \\
\text { COMPLETION } \\
\text { DATE }\end{array}$ & COMMENTS \\
\hline & TASK I - CONTRACT MANAGEMENT \& CLOSE OUT & & & & \\
\hline$\overline{T A}$ & Issue Management Plan for FY95 & Feb 95 & Feb 95 & & Completed \\
\hline TB & Issue Monthly Reports & Apr 95 & As Indicated & & Completed April '95 report \\
\hline \multirow[t]{2}{*}{ IC } & Issue Quarterly Reports & Apr 95 & As Indicated & & Completed Jan-Mar 95 report \\
\hline & TASK $\|$ - FACILITY \& PROPERTY DISPOSITION & & & & \\
\hline$\overline{\pi A}$ & Semi-Annual Government-Owned Property Report & Oct 94 & Oct 94 & & Completed \\
\hline$\overline{11 B}$ & Complete all Engineering Drawings & Dec 94 & Dec 94 & & All drawings completed/updated \\
\hline \multirow[t]{2}{*}{$\overline{\mathrm{IIC}}$} & Annual Government Property Inventory & Dec 94 & Dec 94 & & Completed \\
\hline & TASK III - DATA REPORTING \& ARCHIVING & & & & \\
\hline III A & Identify Data for Archiving & Nov 94 & Nov 94 & & Completed \\
\hline IIIB & Prepare data/documentation for archiving & Feb 95 & Feb 95 & & Completed \\
\hline IIIIC & Issue Superheater/Air Heater Tube Corrosion Studies & & Mar 95 & Sep 95 & \\
\hline IIID & Issue Topical Report - Western Coal Processing System & & Apr 95 & Jun 95 & \\
\hline IIIE & Issue Topical Report - $\mathrm{NO}_{x} / \mathrm{SO}_{x}$ Formation \& Control & & Apr 95 & Jun 95 & \\
\hline \multirow[t]{2}{*}{ IIIF } & Issue Western Coal $1000 \mathrm{Hr}$ POC \& Final Program Summary Report & & May 95 & Jun 95 & \\
\hline & $\begin{array}{l}\text { TASK IV - SITE ENVIRONMENTAL COMPLIANCE \& } \\
\text { REMEDIATION }\end{array}$ & & & & \\
\hline IV A & Complete Removal of Asbestos in the CFFF Cooling Tower & Feb 95 & Mar 95 & & Completed \\
\hline IV B & Install additional groundwater wells for monitoring & & May 95 & Jul 95 & Postponed due to analytical screening \\
\hline IVC & Issue Monthly Water Discharge Monitoring Reports & & As Indicated & & \\
\hline
\end{tabular}




\section{Contract Identification \\ Operation of the MHD Coal-Fired Flow Facility \\ 4. Contractor (Name and Address)}

The University of Tennesses Space Institute

B. H. Goechert Parkway

Tullahoma, Tennessee 37388-8897

\section{Government Funding 9. Contractor Funding}

$\$ 105,842,540.00$

15. Identification Number 16. Reporting Category e.g. contract ine litem or work breakdown structure element)

$\therefore$

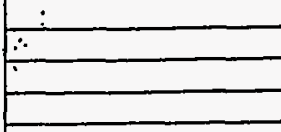

DIRECT LABOR DIRECTLABOR EQUIPMENT

EXPENDABLE MATERIAL OUTSIDE CONTRACTS TRAVEL COMPUTER USAGE

\section{TOTAL DIRECT COSTS} OVERHEAD

GRAND TOTAL

\section{PRELIMINARY}

\begin{tabular}{|l}
$\begin{array}{c}\text { 2. Reporting Period } \\
\text { May i through May 31, } 1995\end{array}$ \\
5. Cost Plan Date \\
February 1995
\end{tabular}

3. Contract Number

DE-AC02-79ET10815

6. Convact Start Date

October 1, 1978

7. Contract Completion Date

September 30, 1995

\begin{tabular}{|l|c|}
\hline $\begin{array}{l}\text { 10. Number of Invoices Billed } \\
\text { Letter of Credit }\end{array}$ & $\begin{array}{c}11 . \text { Frequency } \\
\text { NA }\end{array}$ \\
\hline
\end{tabular}

\begin{tabular}{|l|l|l} 
12. Number of Invoices Paid & 13. Total Invoice Amounts Billed
\end{tabular}

\begin{tabular}{l|l} 
N/A & $\$ 105,316,280.85$ \\
\hline
\end{tabular}

\begin{tabular}{l|l|l}
\hline 17. Accrued Cost & 18. Estimated Accrued Cosis
\end{tabular}

Cash Basis "*

\begin{tabular}{l|l} 
During Reporting Period & Cumulative to Date \\
\hline
\end{tabular}

\begin{tabular}{l|l} 
a. Subsequent & b. Balance of \\
Reporting & Fiscal Year
\end{tabular}

(20)

actual lo Planned c. Actual d Planned

\begin{tabular}{ll|l|l}
\hline 10509567 & b. Planned & c. Actual & d. Planned Jun 95 \\
\hline
\end{tabular}

\begin{tabular}{|l|c|c|c|}
\hline 05095.57 & 114 & 53971,40410 & 54.057 \\
\hline
\end{tabular}

\begin{tabular}{llll}
27 & 1074209837 & 10,765 \\
\hline
\end{tabular}

\begin{tabular}{l|l|l|l|}
\hline & $e$ & 6.037 .946 .54 & 6.038 \\
\hline
\end{tabular}

\begin{tabular}{l|l|l|l|}
2819.26 & 6 & 9.583 .043 .91 & 2.704 \\
\hline
\end{tabular}

\begin{tabular}{l|l|l|l|}
\hline 5.531 .69 & 5 & 7.417 .837 .75 & 7.476 \\
\hline
\end{tabular}

\begin{tabular}{l|l|l|l|}
\hline 808.78 & 1 & 1.018 .464 .94 & 1.022 \\
\hline
\end{tabular}

\begin{tabular}{l|l|l|l|}
\hline 126.94 & 1 & $2,127,728.61$ & 2,129 \\
\hline
\end{tabular}

\begin{tabular}{l|l|l|l|} 
& & & \\
\hline 139.658 .91 & 154 & $90,898,524.22$ & 91,191
\end{tabular}

\begin{tabular}{r|r|r|r|}
\hline $139,658.91$ & 154 & $90,898,524.22$ & 91,191 \\
\hline $24,772.51$ & 27 & $14,417,756,63$ & 14,468 \\
\hline
\end{tabular}

\begin{tabular}{|l|l|l|l|}
\hline & & & \\
\hline $164,431.42$ & 181 & $105,316,280,85$ & 105,659 \\
\hline
\end{tabular}

\begin{tabular}{|c|c|c|c|c|c|}
\hline - & GRAND TOTAL & $164,431.42$ & 181 & $105,316,280.85$ & 105,659 \\
\hline B\&R & AA701030 & 0 & 0 & $65,787.381 .00$ & 65,787 \\
\hline$B \& A$ & AA701035 & 0 & 0 & $516,868.00$ & 517 \\
\hline$B \& A$ & AA701010 & 0 & 0 & $9,187,000.00$ & 9,187 \\
\hline$B \& A$ & AA701020 & 0 & 0 & $179,000.00$ & 179 \\
\hline$B 8 R$ & AA7010105 & 0 & 0 & $26,425,000,00$ & 26,425 \\
\hline $88 \mathrm{~A}$ & AA7010251 & 0 & 0 & $2,000,000,00$ & 2,000 \\
\hline$B \& R$ & AWO & 0 & 0 & $65,000,00$ & 65 \\
\hline$B \& R$ & AWO2 & 0 & 0 & $195,000.00$ & 195 \\
\hline BEA & AWO5 & $164,431.42$ & 181 & $961,031.85$ & 1,304 \\
\hline
\end{tabular}

23. REMARKS: - Ovehead rate effective $7 / 1 / 94$ is $18 \%$ ol TDC less capital equipment, outside contracts ove

.. Actual costs are on cash basis and do not Include outstanding costs ilsted in column 21.

... Includes orders outstanding costs ilsted in column 21.

over $\$ 25,000$, and GRA maintenance fees.

\begin{tabular}{|l|l|l|l|l}
\hline c. Total Contract & $\begin{array}{c}\text { 19. Total Contract } \\
\text { Value }\end{array}$ & 20. Variance & $\begin{array}{c}\text { 21. Unfille } \\
\text { Orders } \\
\text { Outstand }\end{array}$ \\
\end{tabular}

\begin{tabular}{|c|c|c|c|}
\hline 110 & & thru 9/30/95 & \\
\hline
\end{tabular}

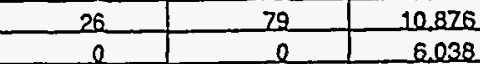

\begin{tabular}{|c|c|c|}
6 & 18 & 2614 \\
\hline 8 & 17 & 7498
\end{tabular}

\begin{tabular}{|c|c|c}
\hline 1 & 17 & 7.498 \\
\hline 0 & 0 & 1.023 \\
\hline
\end{tabular}

\begin{tabular}{|c|c|c|}
\hline & & 2,128 \\
\hline 151 & 442 & 91,697 \\
\hline
\end{tabular}

\begin{tabular}{|c|c|}
\hline & \\
\hline 177 & 519 \\
\hline
\end{tabular}

\begin{tabular}{|c|c|}
\hline 91,697 & 91,347 \\
\hline 14,557 & 14,495 \\
\hline 106,254 & 105,842 \\
\hline 65787 & 65787 \\
\hline 517 & 517 \\
\hline 9,187 & 9,187 \\
\hline 179 & 179 \\
\hline 26,425 & 26,425 \\
\hline 2,000 & 2,000 \\
\hline 65 & 65 \\
\hline 195 & 195 \\
\hline 1,899 & 1.487 \\
\hline
\end{tabular}

$\mid$\begin{tabular}{|l|l|} 
14. Total Payment Rece \\
20. Variance & $\begin{array}{r}\text { 21. Unfilled } \\
\text { Orders } \\
\text { Outstan }\end{array}$
\end{tabular}

\begin{tabular}{l|l}
\hline 7 & 0 \\
\hline 7 & 0 \\
\hline 9 & 0 \\
\hline 5 & 0 \\
\hline & 0 \\
\hline 5 & 0 \\
\hline & 0 \\
\hline
\end{tabular}

\begin{tabular}{|c|r|r|}
\hline 0 & & \\
\hline 0 & 0 & 65,787 \\
\hline 0 & 0 & 517 \\
\hline 0 & 0 & 9,187 \\
\hline 0 & 0 & 179 \\
\hline 0 & 0 & 26,425 \\
\hline 0 & 0 & 2,000 \\
\hline 0 & 0 & 65 \\
\hline 177 & 0 & 195 \\
\hline
\end{tabular}

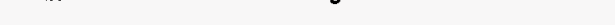$$
\text { (n) }
$$

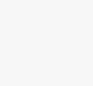




\section{Contract Identification}

Operation of the MHD Coal.Fired Flow Facilly

\section{Contractor (Name and Address)}

The University of Tennesse Space Institute

B. H. Goethert Parkway

Tullahoma, Tennessee 37388-8897

\section{Govemment Funding 9. Contractor Funding}

$\$ 105,842,540.00$

5. Identification Number 16. Reporting Category

(0.9. contract line ltem or work

breakdown structure element)

$\checkmark$

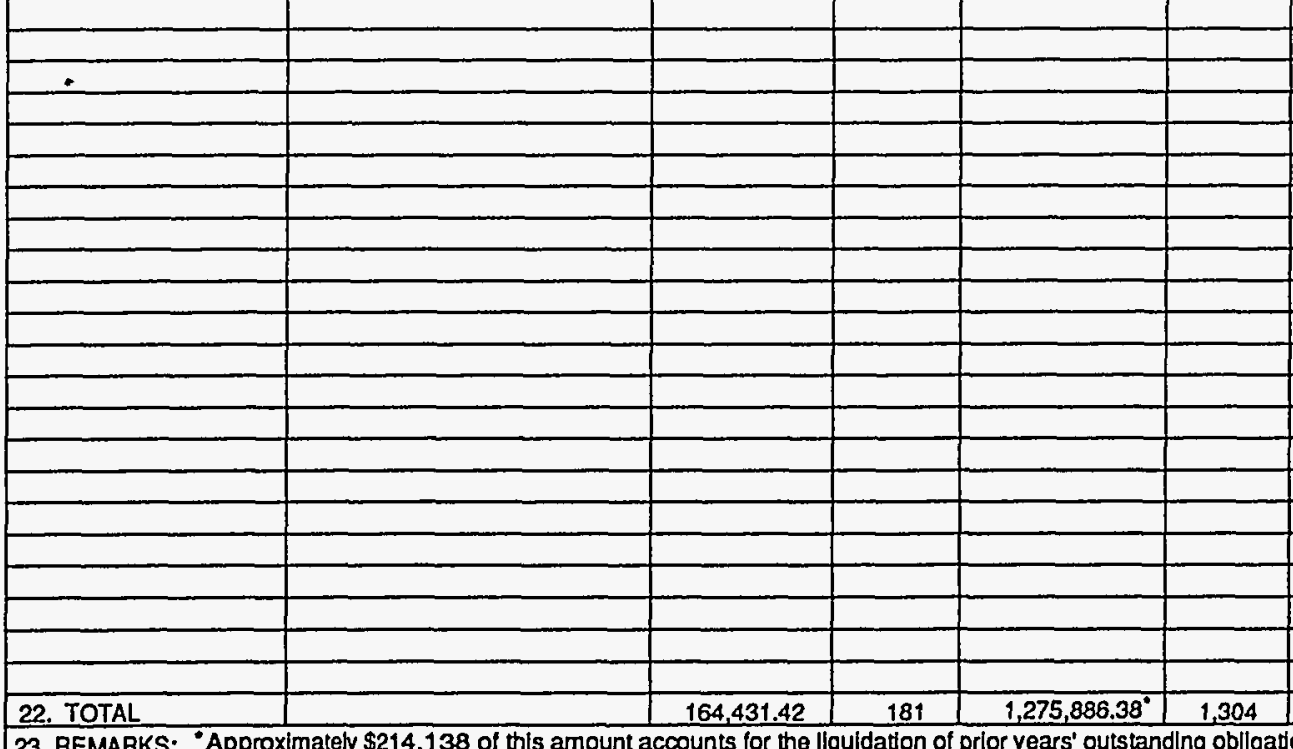

\begin{tabular}{l|l} 
10. Number of Invoices Billed & $\begin{array}{c}11 . \text { Frequency } \\
\text { N/A }\end{array}$
\end{tabular}

Letter of Credit

17. Accrued Cost

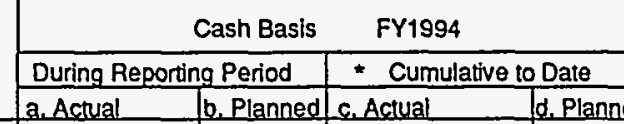

\begin{tabular}{cc|c|c|c|}
\hline Contract ManagemenvCloseout & 30.180 .03 & 34 & 219.704 .51 & 237 \\
\hline
\end{tabular}

\begin{tabular}{l|l|}
\hline Task ! & Contract ManagemenvCloseout \\
\hline Task II & Facilly and Propery Dlsposition
\end{tabular}

Task II

Task I

Data Reporting and Archiving

Slie Environmenta

56,9857

Task IV

23. REMARKS:

Approximately $\$ 214,138$ of this amount
October-December 1994 have been rev

25. Signattre of Contractors Project Manager and Date

Swisn welecan-6/15/95

May 1 through May 31, 1995

5. Cost Plan Date

February 1995

$-60$

\begin{tabular}{llll}
$52,401,95$ & -60 & $410,130,55$ & 422 \\
\hline
\end{tabular}

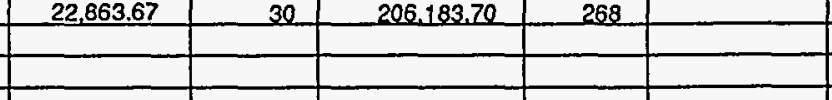

\begin{tabular}{ll|l|l|l|}
\hline & & & & \\
\hline
\end{tabular}

NA

NA

18. Estimated Accrued Costs

. Subsequent $\mid$ b. Balance of

Reportin
Period

Fiscal Year

Adw. Muehihauser, PhD, Administrator
26. SIgnature of Contractors Authorized Financial Representative \& Date
3. Contract Number

DE-AC02-79ET10815

6. Contract Start Date

October 1, 1978

7. Contract Completion Date

September 30, 1995

\begin{tabular}{l|l} 
13. Total Invoice Amounts Billed . & 14. Total Payment Received
\end{tabular} $\$ 105,316,280.85$

\begin{tabular}{|l|l|l|l|}
\hline c. Total Contract & $\begin{array}{l}\text { 19. Total Contract } \\
\text { Value }\end{array}$ & 20. Variance & $\begin{array}{l}\text { 21. Unfilled } \\
\text { Orders } \\
\text { Outstanding }\end{array}$
\end{tabular}

